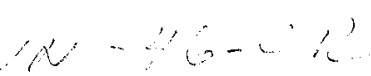

\title{
Topographic Forcing of the Atmosphere and a Rapid Change in the Length of Day
}

David A. Salstein and Richard D. Rosen 


\section{Topographic Forcing of the Atmosphere and a Rapid Change in the Length of Day}

\author{
David A. Salstein and Richard D. Rosen
}

During June to September 1992, a special campaign was held to measure rapid changes in Earth's rotation rate and to relate these measurements to variations in the atmosphere's angular momentum, due principally to changes in zonal winds. A strong rise in both length of day and atmospheric momentum during a particular 6-day subperiod is documented, and this example of a short-period perturbation is identified with a specific regional coupling mechanism. Mountain torques within the southern tropics appear to account for most of the rapid momentum transfer between the solid Earth and atmosphere, with those across South America especially important.

$\mathbf{R}_{\text {ecent }}$ advances in the accuracy of space geodetic techniques used to measure the Earth's rotation, as well as the availability of improved calculations of global atmospheric angular momentum (AAM), have aided the understanding of dynamic interactions among the planet's solid portion, atmosphere, and ocean. On time scales between roughly a fortnight and several years, changes in the angular momentum of the solid Earth, manifested as variations in the length of day (1.o.d.), are almost entirely accounted for by changes in $\operatorname{AAM}(1,2)$. Discrepancies in this balance at high frequencies have been noted, and details of the momentum exchange mechanisms have not been fully understood. To address these issues, a campaign was planned to determine Earth orientation and AAM parameters with the most accurate systems available. This experiment, named SEARCH'92 (3), was conducted from June to September 1992, with a special period of intensive measurements held from 25 July to 8 August.

During the intensive portion of the campaign, values of the relative component of AAM about the polar axis due to winds, $M^{w}$, and of the component due to the

Atmospheric and Environmental Research, Inc., 840 Memorial Drive, Cambridge, MA 02139, USA. planet's mean rotation, $M^{p}$, were computed every 6 hours according to

$$
\begin{aligned}
& M^{\mathrm{w}}=a^{3} g^{-1} \iiint u \cos ^{2} \phi d \lambda d \phi d p \\
& \mathrm{M}^{\mathrm{P}}=a^{4} \Omega g^{-1} \iint p_{\mathrm{s}} \cos ^{3} \phi d \lambda d \phi
\end{aligned}
$$

where $a$ is Earth's mean radius; $g$ is the acceleration due to gravity; $u$ is zonal wind; $\lambda, \phi$, and $p$ are longitude, latitude, and pressure ( $p_{\mathrm{s}}$ is pressure at Earth's surface), respectively; and $\Omega$ is Earth's mean angular velocity. Integrals over the global atmosphere were calculated from operational wind and surface-pressure analyses produced by several of the world's weather centers. These values were collected by the Sub-bureau for Atmospheric Angular Momentum of the International Earth Rotation Service for analysis and distribution (4). During the intensive period of the campaign, AAM appears to undergo a nearly 10-day oscillation superimposed on a (seasonal) upward trend, as shown (Fig. 1A) by estimates from the U.S. National Meteorological Center (NMC) using analyses on a $2.5^{\circ}$ latitude-longitude grid and 12 vertical pressure levels up to $50 \mathrm{mbar}$ (5). Although the oscillatory component is near the high-frequency limit that generally ex- ists now in the measurement of coherent fluctuations between AAM and l.o.d., the changes captured in $\mathrm{M}^{\mathrm{w}}+0.7 \mathrm{M}^{\mathrm{p}}$ (6) during the intensive period explain most $(64 \%)$ of the variance in simultaneous measurements of l.o.d. (7) (Fig. 1A). Most of this agreement is associated with changes in $M^{w}$; during this period, fluctuations in $M^{p}$ contribute little to the variance in 1.o.d. Therefore, study of the NMC daily windbased momentum data further to determine the source and character of momentum variability during the intensive period suffices for a broad understanding of the Earth's rotational behavior during that time as well.

The relative angular momentum of the atmosphere within 46 equal-area latitude belts (8) was calculated from NMC zonal wind analyses for the intensive period. Upon subtraction of the means for that period from all corresponding daily belt values, we derived a time-latitude diagram (Fig. 1B) of the temporal variability of belt momentum anomalies. The relative contribution of each belt to the behavior of $\mathrm{M}^{\mathrm{w}}$ in Fig. $1 \mathrm{~A}$ is shown in Fig. 1C in terms of the fractional covariance between the time series of the individual belt and global $\mathrm{M}^{\mathrm{w}}$ anomalies. Although a strong signal exists near $45^{\circ} \mathrm{S}$, the broad expanse of positive covariance values in low-latitude Southern Hemisphere belts, peaking near $10^{\circ} \mathrm{S}$, indicates that variations there are most responsible for the $\mathrm{M}^{\mathrm{w}}$ fluctuations: The temporal oscillation between negative and positive anomalies at these latitudes mimics the global behavior.

Although Fig. 1 isolates the important centers of momentum variability during our study period, it does not reveal the mechanisms responsible for these variations. The development of a new data set for the two torques that link the atmosphere and Earth (9) allows us an unprecedented opportunity, however, to identify the manner by which the momentum exchange occurs on the time scales dealt with here. One torque 
results from differences in normal pressure forces across mountain barriers, effecting a to the other. The second torque arises from tangential frictional stresses at the atmosphere's lower boundary, which transfer momentum with the land or ocean below. If applied over the ocean, this stress can be transmitted within a day or so to the solid Earth through the development of sea-level differences at the continental margins on opposite sides of a basin, as the ocean adjusts barotropically to the wind-forcing field (10).

Global values of these two torques have been computed four times per day for the intensive period of SEARCH'92 as part of an ongoing effort at NMC (11). Mountain torque terms are calculated from the surface pressure and topography fields, whereas frictional stress torques are calculated from cast model (12). Global values averaged daily of these two torques are displayed in Fig. 2. Mountain torques are positive during most of the period and dominate the frictional stress term, which is negative and relatively steady throughout (13). The sum of the mountain and stress torques should, in principle, be identical to the time derivative of global momentum. The agreement in Fig. 2B, though very good, is not perfect because momentum is computed from the wind and pressure analyses of the NMC system, whereas the torque calculations involve a parameterization of friction and other approximations, such as the use of discrete temporal representations. transfer of momentum from one component the physical parameterizations of the fore-

To study the character of the torques in greater detail, we focus now on the 6-day period from 31 July through 5 August, when the total torque was at its peak, corresponding in Fig. 1A to the time of the most rapid rise in AAM. Of the two torques, only the mountain one is positive during this 6-day period, and it determines the sign of the total torque. Hence, the acceleration experienced by the global atmosphere during this period must be due to interactions with Earth's topography. Therefore, we examine the mountain torque more closely by decomposing its global mean value for 31 July to 5 August into contributions made at individual latitudes (Fig. 3A). During this period, the main contribution to the global mountain torque appears to come from the region between the equator and $30^{\circ} \mathrm{S}$ (14).

The torque data set now available permits us to isolate, in turn, which portions of the $0^{\circ}$ to $30^{\circ} \mathrm{S}$ region are most responsible for the maximum there, and we do so by dividing the region into three longitudinal sectors on the basis of the distribution of continents. The net mountain torque across a landmass is generally the small difference between large values of opposite sign; its evaluation, therefore, involves a sensitive calculation $(15,16)$. For the 6 -day period of rapid atmospheric acceleration studied here, southern tropical Africa and South America dominate (17) the transfer of momentum from the solid Earth to the atmosphere both within the Southern Hemisphere tropics and, therefore from Fig. $3 \mathrm{~A}$, for the globe as a whole.

Fig. 1. (A) Comparison of relative plus planetary AAM (thick solid trace) and of relative momentum alone (thin solid trace) with the length of day during the intensive period of SEARCH'92. The atmospheric series are computed from once daily (00 UTC) NMC analyses of surface pressure and winds to the 50-mbar level and are reported here in momentum units along the left as well as equivalent l.o.d. units along the right, with the planetary component of momentum weighted by a 0.7 factor relating to the Earth's response. The l.o.d. values (dotted trace) are based on Very Long Baseline Interferometry measurements. Means have been removed from all series. The straight dashed lines in this and the next figure denote the period during which the
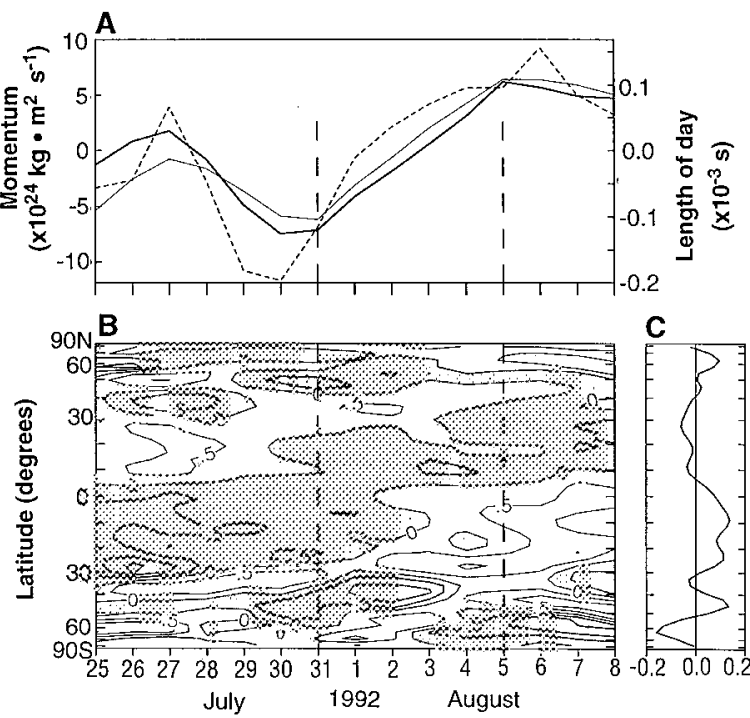

atmosphere underwent a rapid acceleration and that we focus on to determine the mechanism causing the acceleration. (B) Time-latitude diagram of the difference between the relative angular momentum within 46 equal-area latitude belts during the intensive period of SEARCH'92 and the mean for each belt during the period. Values, given in $10^{24} \mathrm{~kg} \cdot \mathrm{m}^{2} \mathrm{~s}^{-1}$, are based on once daily (00 UTC) NMC winds to 50 mbar. Easterly (negative) anomalies are shaded. (C) Fractional covariance of anomaly belt momentum values with $M^{\mathrm{w}}$, the global sum of the values during the intensive period. The sum of the 46 numbers comprising the curve is unity.
The torques over South America in particular appear to play a special role in the explanation of much of the behavior of the globally integrated mountain torque during the 31 July to 5 August study period. This result is evident from Fig. 3B, in

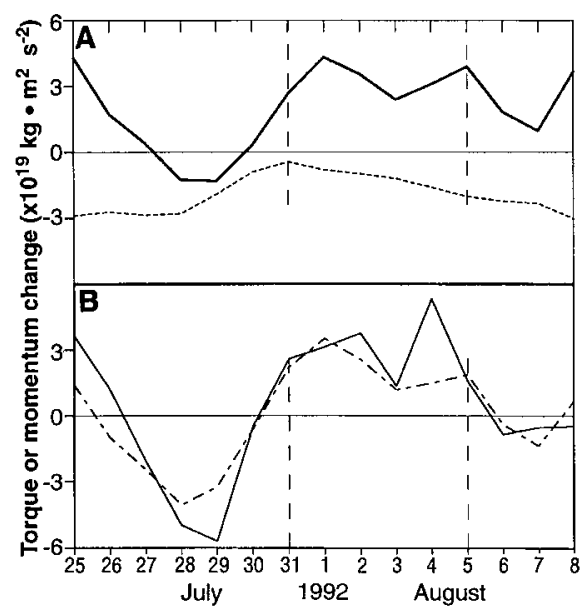

Fig. 2. (A) Daily means of mountain (solid trace) and friction (dashed trace) torques from the NMC analysis-forecast system during the intensive period of SEARCH'92. (B) Time derivative of the relative plus planetary AAM (solid trace) compared with the sum of mountain and friction torques (dashed trace)

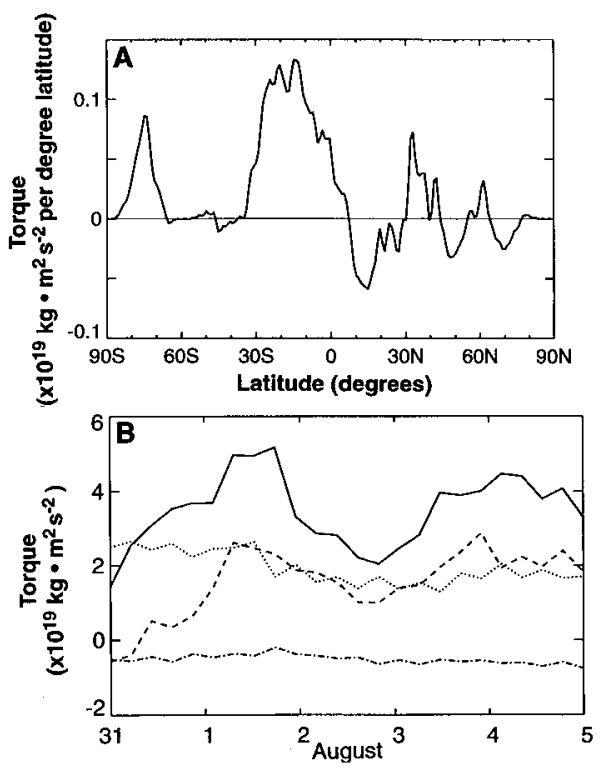

Fig. 3. (A) Profile in latitude of the zonal mean mountain torque averaged during 31 July to 5 August 1992. The integral under the curve equals the mean global mountain torque over the period, that is, the mean for the mountain torque curve in Fig. 2 over these 6 days. (B) Four times daily series of mountain torque for the globe (solid trace) and for three continental regions in the $0^{\circ}$ to $30^{\circ} \mathrm{S}$ band during 31 July to 5 August 1992: South America (thick dashed trace), Africa (thin dotted trace), and East Indies-Australia (mixed dotted and dashed trace). 
which we have plotted time series of the global mountain torque and the torque across continental portions of the $0^{\circ}$ to $30^{\circ} \mathrm{S}$ belt for this period. Although the torque across the southern tropical portion of Africa is on average larger than that across tropical South America, the latter is much better correlated in time with the global torque, explaining $69 \%$ of the variance in the global time series during these 6 days. Indeed, when this comparison is extended to all of South America, this proportion rises to $88 \%$. Fluctuations in torque with time determine the shape of temporal variations in angular momentum, so from this perspective conditions around South America during 31 July to 5 August 1992 were especially important in determining the character of AAM then.

The relative importance of mountain versus friction torques in the planetary momentum cycle appears to be a function of time scale (18), and the dominance of the former in our case study is consistent with other studies of short-period changes in AAM. Thus, the mountain torque was more responsible for the variations observed in AAM within the 2-month special observing periods of the 1979 Global Weather Experiment (16) and explains much of the momentum anomaly observed at the height of the El Niño-Southern Oscillation event in January 1983 (19). Torques over land at submonthly periods are more important than those over the ocean, according to a recent study (20) that included satellite-based measurements of ocean stresses, and multiyear simulations of the atmosphere's general circulation (21) support this result. The present case study, of course, illustrates an instance when the mountain torque across a single continent is highlighted.

Synoptic maps of mean sea-level pressure based on NMC analyses reveal that a strong high-pressure center moved to the east of the Andes near the beginning of August 1992, a shift that appears to account for the acceleration of the atmosphere then. A strong zonal pressure gradient associated with this progression became centered over the spine of the Andes on 1 August (Fig. 4), corresponding to the increase in the acceleration of the atmosphere that began on that day (Fig. 1). Investigation of the role of other synoptic events in the global momentum budget will be part of a more general study of "1- to 2-week" waves in angular momentum evident throughout much of our record. In these studies, the manner in which various time scales of behavior are separated can be important in the interpretation of results. For example, when trends are also removed from the belt momentum series in Fig. 1B, so as to isolate a 10-day fluctuation, variations near $40^{\circ} \mathrm{S}$ become much more crucial for global $\mathrm{M}^{\mathrm{w}}$ variations than those near $10^{\circ} \mathrm{S}$ (as in Fig. 1C). Similarly, when mean torques for the period are removed (equiv alent to the detrending of AAM), frictional torques over $40^{\circ} \mathrm{S}$ must be invoked to help account for the rising portion of the AAM "wave," and contributions from mountain torques over several regions besides those highlighted here become more important.
Fig. 4. Maps of sea-level pressure, in millibars, based on NMC analyses in an area near South America for 00 UTC on (A) 31 July and (B) 1 August 1992, just before and during the peak acceleration of the atmosphere. A high-pressure system moves just to the east of the Andes, resulting in a strong zonal pressure gradient across the mountainous topography

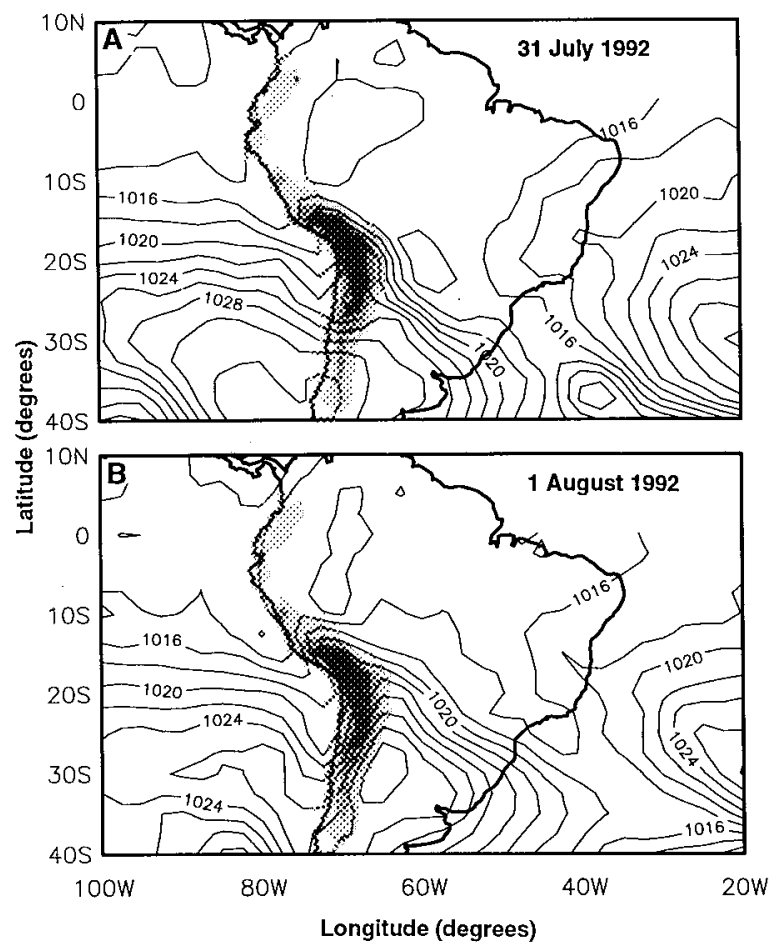

\section{REFERENCES AND NOTES}

1. R. D. Rosen, D. A. Salstein, T. M. Wood, J. Geophys. Res. 95, 265 (1990); R. Hide and J. O. Dickey, Science 253, 629 (1991).

2. Contributions of the oceans and core are small at these time scales; those due to external tides can be significant, but because they occur at specific, well-known frequencies, they are easily isolated and so are removed here

3. J. O. Dickey, Adv. Space Res. 13, 185 (1993)

4. D. A. Salstein, D. M. Kann, A. J. Miller, R. D. Rosen, Bull. Am. Meteorol. Soc. 74, 67 (1993).

5. Close agreement among AAM values from all the meteorological centers helps to justify the use of a single AAM series here. Although higher frequency data are available, the once daily values shown in the figure are appropriate for representation of the oscillation of interest.

6. The 0.7 factor applied to the pressure term involves a Love number correction to account for the response of the nonrigid, solid Earth to atmospheric loading.

7. The measurement technique for the determination of Earth's rotation here is Very Long Baseline Interferometry [D. S. Robertson, W. E. Carter, J. Campbell, H. Schuh, Nature 316, 424 (1985)].

8. R. D. Rosen and D. A. Salstein, J. Geophys. Res. 88,5451 (1983)

9. G. H. White, in "Proceedings of the American Geophysical Union Chapman Conference on Geodetic VLBI: Monitoring Global Change," Nat. Oceanic Atmos. Adm. Tech. Rep. NOS 137 NGS 49 (1991), p. 262

10. R. M. Ponte, J. Geophys. Res. 95, 11369 (1990).

11. G. H. White, in "Research Activities in Atmospheric and Oceanic Modeling." CAS/JSC Work. Group Numer. Exp. Rep. no. 18 (World Meteorological Organization, Geneva, 1993), 2.3-2.4

12. In the NMC model, surface momentum exchange is calculated with a bulk aerodynamic formula in which the exchange is proportional to the vertical momentum gradient, with the proportionality dependent on wind speed and surface layer static stability [M. Kanamitsu, Wea. Forecasting 4, 335 (1989)].

13. Friction torques over both ocean and land are each negative, with mean values of $-0.53 \times 10^{19}$ and $-1.46 \times 10^{19} \mathrm{~kg} \cdot \mathrm{m}^{2} \mathrm{~s}^{-2}$, respectively, during the intensive period.

14. Substantial frictional torques also transfer mo* mentum into the Southern Hemisphere tropical atmosphere, but these are more than compensated by negative frictional torques in the southern extratropics.

15. J. M. Wahr and A. H. Oort, J. Atmos. Sci. 41, 190 (1984).

16. R. Swinbank, Q. J. R. Meteorol. Soc. 111, 977 (1985)

17. Mountain torques computed for southern tropical portions of Africa, South America, and East Indies-Australia are $1.94 \times 10^{19}, 1.54 \times 10^{19}$, and $-0.52 \times 10^{19} \mathrm{~kg} \cdot \mathrm{m}^{2} \mathrm{~s}^{-2}$, respectively, based on the division of the $0^{\circ}$ to $30^{\circ} \mathrm{S}$ region into sectors at $0^{\circ}, 90^{\circ} \mathrm{E}$, and $120^{\circ} \mathrm{W}$ longitudes.

18. R. D. Rosen, Surv. Geophys, 14, 1 (1993).

19. W. L. Wolf and R. B. Smith, J. Atmos. Sci. 44, 3656 (1987)

20. R. M. Ponte and R. D. Rosen, J. Geophys. Res. 98, 7317 (1993).

21. G. J. Boer, ibid. 95, 5511 (1990).

22. We thank G. White of the NMC and D. Robertson of the National Ocean Service for supplying torque and I.o.d. data for this study, D. Kann for valuable help in preparing the AAM data for this period, and $K$. Cady-Pereira and $P$. Nelson of Atmospheric and Environmental Research, Inc., for providing excellent programming support. Supported by NASA under both its Geophysics Program (contract NASW-4751) and Earth Observing System Program (contract NAGW-2615). 